Präv Gesundheitsf 2021 · 16:193-199

https://doi.org/10.1007/s11553-021-00852-x

Eingegangen: 9. Januar 2021

Angenommen: 12. April 2021

Online publiziert: 4. Mai 2021

(c) Der/die Autor(en) 2021

\section{Gabriele Buruck' · Sophia Haitsch ${ }^{2}$}

'Professur für Gesundheitsförderung und Prävention, Westsächsische Hochschule Zwickau, Zwickau, Deutschland

${ }^{2}$ Employer Branding und Talentmanagement, SachsenEnergie AG, Dresden, Deutschland

\title{
Emotionale Kompetenzen und psychische Gesundheit
}

\section{Eine Querschnittstudie in Gesundheitsberufen}

In Gesundheitsberufen stellt der Umgang mit den eigenen Emotionen eine berufliche Anforderung dar, denn hier ist der Mensch der Arbeitsgegenstand. Dabei kann es vorkommen, dass die Beschäftigten mit Personen in Verbindung treten, die ihnen unsympathisch sind oder dass die Interaktion an sich negative Gefühle verursacht. Trotzdem sind Beschäftigte durch bestimmte sog. Darstellungsregeln angehalten, sich auch in solchen Situationen freundlich zu geben oder wenigstens ihren Unmut nicht offen $\mathrm{zu}$ zeigen.

\section{Emotionsarbeit und Emotions- regulation}

Diese Darstellungsregeln werden durch das eigene berufliche Verständnis und durch den Arbeitgeber definiert. Emotionsarbeit, welche die Umsetzung dieser Regeln durch das Regulieren der eigenen Emotionen und damit der eigenen

Die Daten der ersten Stichprobe wurden im Rahmen eines Interventionsprojekts für die stationäre Altenpflege in Sachsen („CURS Psychische und körperliche Fehlbelastung \& chronischer unspezifischer Rückenschmerz in der Altenpflege") erhoben. Die Datenerhebung erfolgte im Zeitraum von 2012 bis 2015 als Trendstudie.

Die zweite, kleinere Stichprobe umfasste 144 Beschäftigte im Rettungsdienst. Sie wurde im Rahmen der Forschungsprojekte "Stressregulierung als Primärprävention bei Auszubildenden zum Notfallsanitäter" und "Transfer Training emotionaler Kompetenzen (TEK) - Schnittstelle Praxisanleiter" untersucht. Die Datenerhebung fand im Zeitraum 2014 bis 2017 als Trendstudie statt.
Handlung beinhaltet, betrifft somit alle Arten von zwischenmenschlichen Interaktionen bei der Arbeit [19, 30]. Beide Anforderungen, die Regulation der Emotionen und die kognitiv-psychische Regulation der Arbeitstätigkeit, wirken sich auf das psychische und körperliche Erleben und damit die Gesundheit aus [20, 29]. Als Folge der Emotionsarbeit können drei emotionale Dimensionen identifiziert werden: Die geforderte, die gefühlte und die gezeigte Emotion [30]. Alle drei Dimensionen werden in Situationen aktiviert, in denen es zu einem Erleben einer emotionalen Regeldissonanz kommt [30]. Nach Zapf et al. [30] definiert sich diese emotionale Regeldissonanz durch die Häufigkeit und Intensität von Situationen, bei denen positive Darstellungsregeln gefordert, innerlich aber nicht gefühlt werden. Folglich muss die tatsächlich erlebte Emotion unterdrückt werden, um die geforderte zeigen zu können. Das bedeutet, dass bei Interaktionen mit anderen Menschen, bei denen durch Regeln bestimmte Emotionsdarstellungen verlangt werden, immer ein innerer Soll-Ist-Abgleich geschieht. Wird als Ergebnis eine Bedrohung der eigenen Ziele wahrgenommen, weil die gefühlte Emotion nicht mit der geforderten Emotion übereinstimmt, entsteht bei dem Beschäftigten langfristig ein innerer emotionaler Konflikt. Dieser kann durch das Fehlen von konstruktiven emotionalen Bewältigungsstrategien weiter verschärft werden.

Als Strategien der Emotionsregulation wurden bisher überwiegend das
Oberflächenhandeln („surface acting“ - Anpassung eines Emotionsausdrucks) und Tiefenhandeln („deep acting“ - Verändern des emotionalen Erlebens) mit Bezug auf Indikatoren von Gesundheit wissenschaftlich untersucht [26]. Vor allem das Befolgen der Darstellungsregeln, aber auch das Erleben von emotionaler Regeldissonanz gehen mit erhöhter emotionaler Erschöpfung einher [16, 26]. In der Bundesrepublik leiden rund 4,2\% der Erwachsenbevölkerung unter einem Burnout-Syndrom [15], wobei Frauen mit 5,2\% häufiger unter dem chronischen Erschöpfungssyndrom leiden. Präzise Angaben zur Prävalenz (Häufigkeit) von Burnout in Deutschland sind aufgrund des Fehlens von einheitlichen Diagnosekriterien aber nicht möglich [22].

In dem wissenschaftlichen Modell von Grandey und Melloy [17] wird der bisherige Forschungsstand der Emotionsarbeit erstmalig mit Konzepten der Emotionsregulation zusammengeführt. Ausgelöst durch ein Arbeitsereignis wird der Beschäftigte in die Situation versetzt, seine eigenen Emotionen zu regulieren. Ein erfolgreiches Gelingen oder Scheitern entscheiden darüber, ob sich für die Person oder ihren Interaktionspartner Wohlbefinden im Sinne einer Zielerreichung einstellt [17].

\section{Konstruktiver Umgang mit Emotionen}

Sinnvoll erscheinen an dieser Stelle Interventionen, welche allgemeine emotiona- 


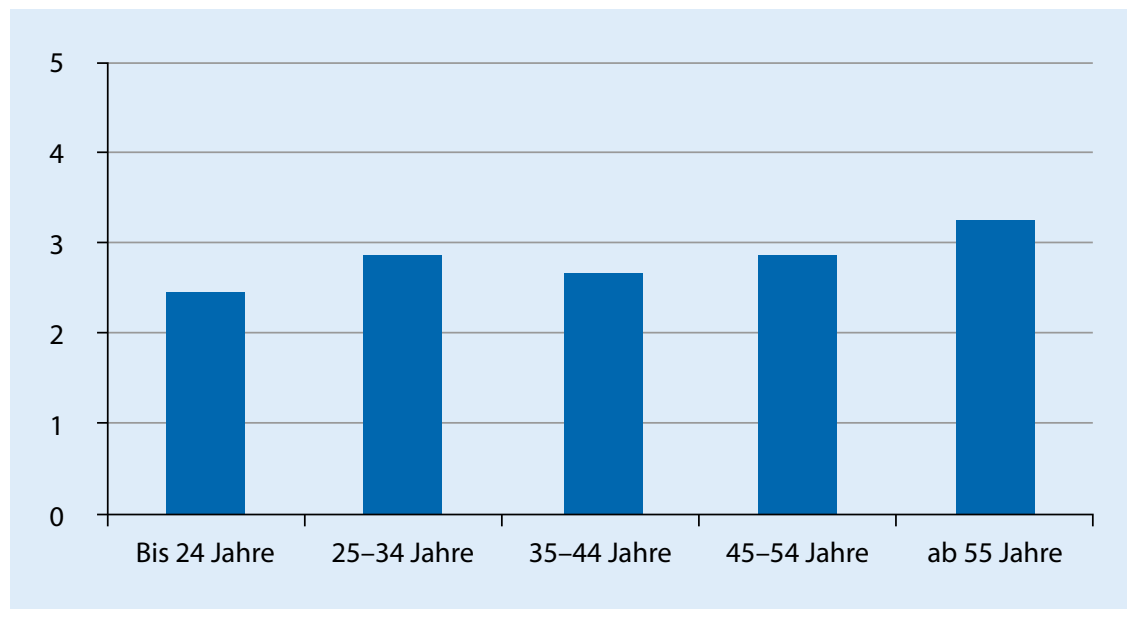

Abb. 1 A Ausprägung des eingeschätzten Wohlbefindens am Medianwert. (Darstellung der Ergebnisse WHO5)

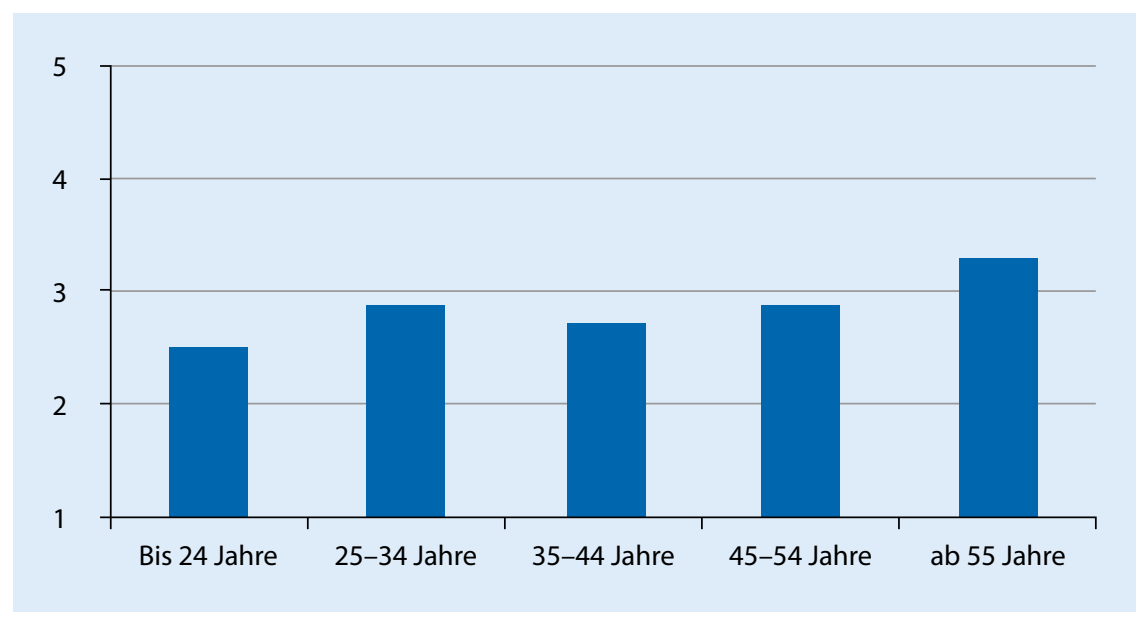

Abb. 2 \ Ausprägung des emotionalen Erschöpfung als eine Burnout-Dimension am Medianwert. (Darstellung der Ergebnisse aus dem Maslach Burnout Inventory - General survey [MBS-GS-D])

le Kompetenzen trainieren (z. B. Training emotionaler Kompetenzen - TEK, [2]), um durch eine hohe Variabilität alle Dimensionen von Emotionsarbeit zu stärken [5, 8-11]. Hierbei stellt insbesondere die Kompetenz des Regulierens der eigenen Emotionen den Kern konstruktiver Emotionsregulation dar [2]. Verändert werden kann dabei die Qualität, die Intensität und/oder die Dauer einer Emotion. Die Voraussetzungen für eine konstruktive Emotionsregulation sind erfüllt, wenn Individuen dazu in der Lage sind, die eigenen Emotionen zielgerichtet zu beeinflussen, $d$. h. sie zu regulieren. Für die psychische Gesundheit ist diese Fähigkeit von zentraler Bedeutung.

Aus diesen Vorüberlegungen leiten sich die Studienziele ab, welche den Zusammenhang von arbeitsbezoge-
Buruck et al. [7] und Buruck und Dörfel [8].

\section{Beschreibungen der Stichprobe}

Die Betrachtung von Altersunterschieden ist in emotional-kognitiven Prozessen der Emotionsarbeit relevant, wobei hier noch offene Fragen zum Einfluss und der Wirkung des Alters ausstehen [25]. Für die deskriptive Datenanalyse wurde der Median der relevanten Konstrukte ermittelt (• Abb. 1, 2 und 3).

Die Fragen nach dem subjektiven psychischen Wohlbefinden wurden folgendermaßen beantwortet: Die Beschäftigten in der stationären Altenpflege und dem Rettungsdienst schätzen ihr Wohlbefinden über alle Alterskategorien als überwiegend gut ein $(M W=2,76$; $S D=1,06$ auf einer Skala $0-5$; entspricht etwas mehr als die Hälfte der Zeit). Je höher die Alterskategorie, umso besser wird das psychische Wohlbefinden wahrgenommen (ab 55 Jahre, $M W=2,97 ; S D=1,17)$. Die Ergebnisse sind in Abb. 1 dargestellt.

Weiterhin wird die selbsteingeschätzte emotionale Erschöpfung berichtet. Wie in - Abb. 2 dargestellt, gaben die Beschäftigten an, eine mittlere bis erhöhte emotionale Erschöpfung in Bezug auf ihre Arbeit zu empfinden. Der Mittelwert von 3,62 entspricht der Aussage, dass sich die Studienteilnehmer mehrmals im Monat emotional erschöpft fühlen. Insgesamt gaben $38 \%$ der Teilnehmer an, bis zu einmal im Monat emotionale Erschöpfung zu erleben. Die restlichen $62 \%$ berichteten, sich mehrmals im Monat emotional erschöpft zu fühlen (18\% sogar einmal in der Woche und häufiger). Die Personen der Alterskategorie ab 55 Jahre, welche knapp $10 \%$ der Gesamtstichprobe ausmachen, berichten die höchsten Werte an emotionaler Erschöpfung $(M W=3,91 ; S D=1,47)$.

Um das Ausmaß der erlebten quantitativen Arbeitsanforderungen (Arbeitstempo, Zeitdruck, Zeitumfang und Aufgabenmenge), aber auch der Ressource Spielräume (Ausführbarkeit, Autonomes Arbeiten und Mitbestimmung bei Entscheidungen) zu ermitteln, schätzten die Beschäftigten diese Arbeitsmerkmale auf einer Skala von 1-5 ein (1 entspricht hier- 
bei jeweils der niedrigsten, 5 der höchsten Ausprägung der Erhebungsvariable in diesem Spektrum).

Die Beschäftigten beurteilten die Arbeitsanforderungen in Bezug auf Intensität und Zeit als gering bis mittel $(M W=3,0 ; S D=0,79)$. Die erlebten Spielräume wurden allerdings auch im geringen bis mittleren Bereich angegeben $(M W=2,89 ; S D=0,82)$. Die Ergebnisse sind in - Abb. 3 dargestellt.

Bezüglich der emotionalen Kompetenz (Regulieren) bewegen sich die Angaben der Personen überwiegend in einem eher niedrigen Bereich $(S W=7$; $S D=2,16)$ und sind somit eher unterdurchschnittlich ausgeprägt (• Abb. 4). Bei fast der Hälfte der Befragten ergaben die Skalenwerte einer geringen emotionalen Kompetenz, bei ca. $30 \%$ konnten durchschnittliche Kompetenzen ermittelt werden und lediglich $20 \%$ weisen überdurchschnittliche Werte auf. Das bedeutet, es wird von den meisten Beschäftigten manchmal $(M W=2,30)$ die Kompetenz angewandt, welche durch die 3 Items („In der letzten Woche ... konnte ich positive Gefühle gezielt herbeiführen; konnte ich meine negativen Gefühle beeinflussen; war mir klar, dass ich meine Gefühle beeinflussen kann") beschrieben wird. Die Normwerte, mit denen die Ergebnisse dieser Stichprobe verglichen wurden, orientieren sich an Normwerten der Stichproben, welche durch die Arbeitsgruppe von Prof. Matthias Berking ermittelt wurden (http://www.psyprasoft.de/EmoCheck. html, [6]). Hierbei werden für die einzelnen Kompetenzen Summenwerte $<7$ als unterdurchschnittlich bezeichnet, durchschnittliche Ausprägungen sind durch Summenwerte von 7-9 gekennzeichnet. Wird ein Summenwert von 10-12 erreicht, so spricht man von sehr hohen Kompetenzen.

\section{Zusammenhänge von Emotionsregulation und Wohlbefinden}

Um vermutete Beziehungen zwischen den Konstrukten zu überprüfen, wurden bivariate Korrelationen berechnet. Die entsprechenden Korrelationskoeffizienten geben Aufschluss über den Grad der

Präv Gesundheitsf 2021 · 16:193-199 https://doi.org/10.1007/s11553-021-00852-x

(c) Der/die Autor(en) 2021

\section{G. Buruck · S. Haitsch}

\section{Emotionale Kompetenzen und psychische Gesundheit. Eine Querschnittstudie in Gesundheitsberufen}

\section{Zusammenfassung}

Hintergrund. Im Jahr 2020 waren 5,7 Mio. Menschen in Gesundheits- und Pflegeberufen tätig. Der Kontakt mit anderen Menschen ist in diesen Berufen Teil des Arbeitsauftrags und der Mensch ist der Arbeitsgegenstand.

Ziel der Arbeit. Das Ziel der Arbeit ist die Untersuchung der Auswirkung von psychischer Belastung (Arbeitsintensität, Spielräume), emotionaler Erschöpfung und emotionalen Kompetenzen (Regulation) auf das psychische Wohlbefinden bei Beschäftigten in Gesundheitsberufen. Material und Methoden. Die Erhebung der Querschnittstudie von 624 Beschäftigten aus der Altenpflege und dem Rettungsdienst (72,5\% weiblich) erfolgte im Rahmen zweier Projekte zur betrieblichen Gesundheitsförderung. Zum Einsatz kamen standardisierte validierte Verfahren, welche mittels Korrelationsanalysen sowie einer hierarchischen Regressionsanalyse zur
Vorhersage des psychischen Wohlbefindens ausgewertet wurden.

Ergebnisse. Hohe Arbeitsintensität, hohe Spielräume, hohe emotionale Erschöpfung und eine hohe emotionale Kompetenz (Regulation) tragen zur Aufklärung des psychischen Wohlbefindens bei $\left(R^{2}=33 \%\right)$. Schlussfolgerung. Anhand der Ergebnisse wird deutlich, dass neben dem Erleben von Arbeit und Gesundheit auch emotionale Kompetenzen einen Einfluss auf die psychische Gesundheit bei Beschäftigten in Gesundheitsberufen haben. Damit leistet die Studie einen wichtigen Beitrag für die Entwicklung von Maßnahmen des betrieblichen Gesundheitsmanagements in diesen Berufsgruppen.

\section{Schlüsselwörter}

Emotionale Kompetenzen - Psychische Gesundheit · Altenpflege - Rettungsdienst · Emotionsregulation - Wohlbefinden . Emotionale Erschöpfung

\section{Emotional skills and mental health. A cross-sectional study in health professions}

\section{Abstract}

Background. In 2020, 5.7 million people worked in health and nursing professions in Germany. In these professions, close contact with other people is a fundamental part of the work and human beings are often the object of the work itself.

Objective. The goal is to examine the impact of psychological strain (work intensity, job decision latitude), emotional exhaustion and emotional competencies (regulation) on the mental well-being of employees in healthcare and nursing professions.

Materials and methods. The cross-sectional study with 624 employees working in eldercare or emergency medical services ( $72.5 \%$ female) was conducted in the context of two projects on workplace health promotion. We used validated procedures that contributed to predict the individual mental well-being via correlation analysis and a hierarchical regression analysis.
Results. High levels of work intensity, job decision latitude, emotional exhaustion and emotional competencies (regulation) contribute to explain mental well-being $\left(R^{2}=33 \%\right)$.

Conclusion. The results indicate that, besides experiencing work and health, emotional competencies have an impact on the mental health of employees in health professions. Consequently, this study makes an important contribution to the development of measures of workplace health management in these occupational groups.

\section{Keywords}

Emotional competencies - Mental health . Eldercare - Emergency medical services . Emotion regulation . Well being $\cdot$ Emotional exhaustion 


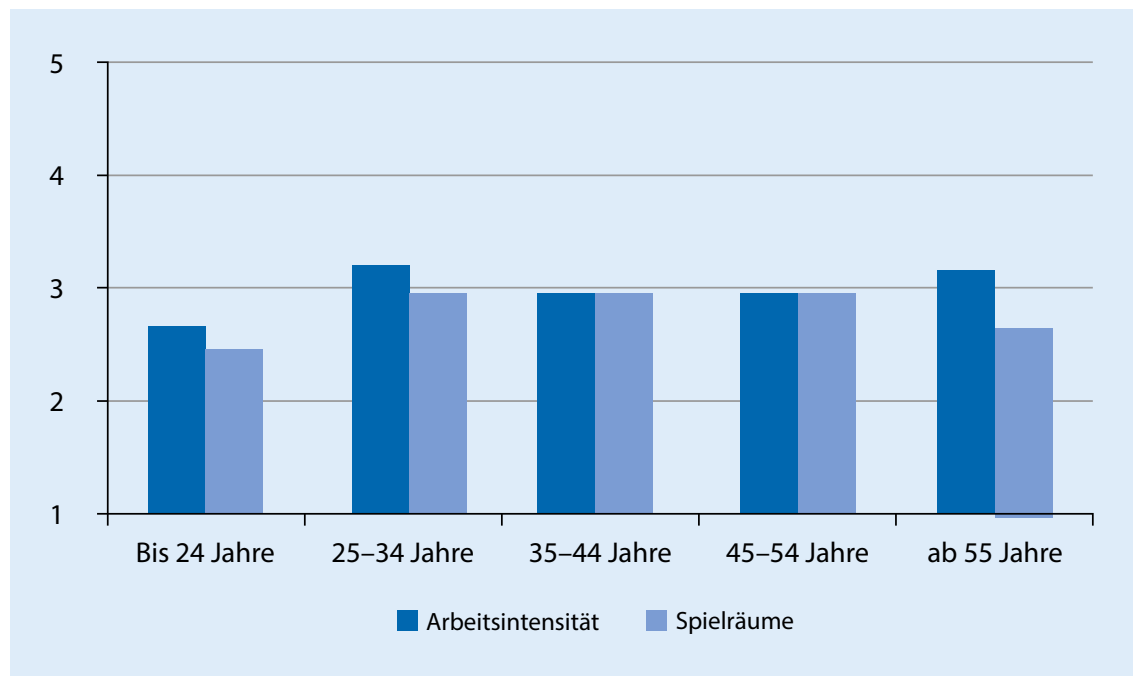

Abb. $3 \Delta$ Ausprägung der eingeschätzten Arbeitsanforderungen und Ressourcen am Medianwert. (Darstellung der Ergebnisse aus den Aspekten des Berufslebens [AWS] und dem Kopenhagen Psychosozialen Fragebogen [COPSOQ])

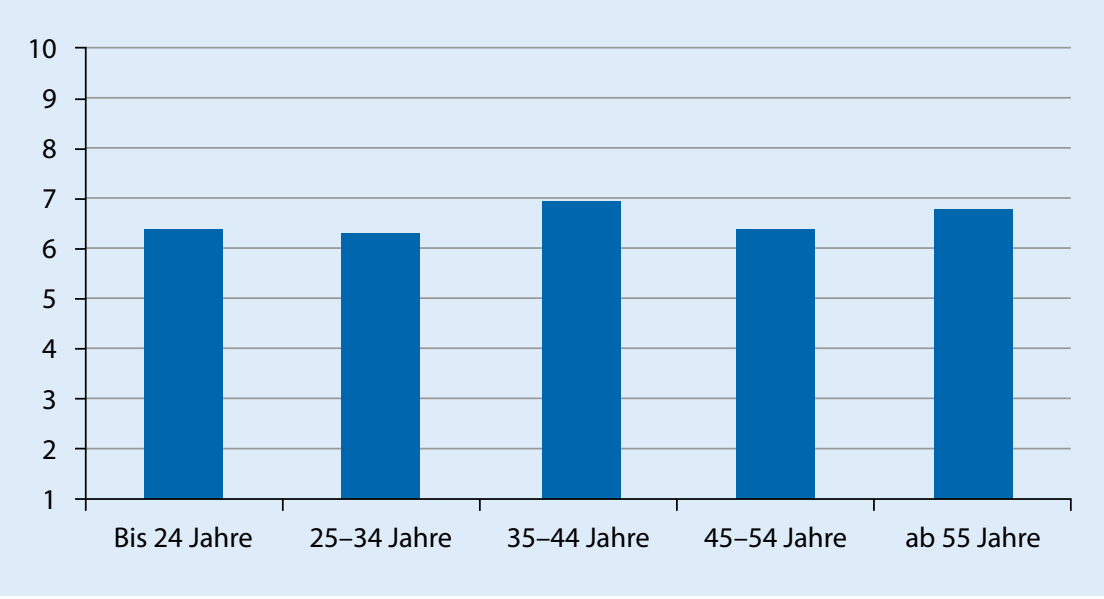

Abb. $4 \Delta$ Ausprägung der emotionalen Kompetenz anhand des Summenwertes. (Darstellung der Ergebnisse aus dem Fragebogen zur Selbsteinschätzung emotionaler Kompetenzen [SEK-27])

linearen Abhängigkeit zwischen zwei Variablen, indem sie die Richtung und Stärke des Zusammenhangs quantifizieren [12]. Eine Korrelation von $|r s|>0,10$ lässt auf einen schwachen Zusammenhang schließen, $|r s|>0,30$ auf einen mittleren und $|r s|>0,50$ auf einen starken Zusammenhang [13]. Die Wahl des Korrelationskoeffizienten ist abhängig vom vorliegenden Datenniveau und der -verteilung [12, 14]. Da die Normalverteilungsannahme für alle Modellvariablen bis auf die psychischen Arbeitsmerkmale abgelehnt wurde, wurde der Rangkorrelationskoeffizient $r s$ nach Spearman verwendet (•Tab. 1). schen den Angaben zur emotionalen Erschöpfung und der emotionalen Kompetenz (Regulieren) besteht ein mittlerer negativer Zusammenhang. So berichten die Personen mit niedriger emotionaler Kompetenz höhere Werte der emotiona- der Ursprungsdatensätze als Kontrollvariable ergibt sich vor allem mit dem Alter ein starker negativer Zusammenhang.

\section{Einflussfaktoren auf das Wohlbefinden und emotionale Kompetenz}

Die auf den Korrelationsanalysen basierenden Ergebnisse bilden die Grundlage für die hierarchische Regressionsanalyse mit den Kontrollvariablen (Geschlecht, Alter und Ursprungsdatensätze). Die Voraussetzungen für eine hierarchische Regression wurden vorab geprüft. Die Ziele der Studie postulierten:

- einen negativen Zusammenhang der Arbeitsintensität mit dem psychischen Wohlbefinden,

- einen positiven Zusammenhang der Spielräume mit dem psychischen Wohlbefinden,

- einen negativen Zusammenhang der emotionalen Erschöpfung mit psychischem Wohlbefinden sowie - einen positiven Zusammenhang der emotionalen Kompetenz mit hohem Wohlbefinden.

Mit anderen Worten, es sollte getestet werden, ob niedrigere Stressoren und höhere Ressourcen sowie eine höhere emotionale Kompetenz mit besserer psychischer Gesundheit einhergehen. Die Ergebnisse aus - Tab. 2 zeigen den Einfluss der Prädiktoren (psychische Arbeitsmerkmale, Burnout und emotionale Kompetenz) unter Einbeziehung der Kontrollvariablen.

Alle im Modell enthaltenen Variablen haben einen signifikanten Einfluss auf das Wohlbefinden Insgesamt klärt das Regressionsmodell $33 \%$ der Varianz für das psychische Wohlbefinden auf, wobei v.a. die Aufnahme der emotionalen Erschöpfung in das Modell die Varianzaufklärung signifikant erhöhte $\left(\mathrm{R}^{2}=0,28\right.$; $\left.\mathrm{R}^{2}{ }_{\text {korr }}=0,27 ; \mathrm{F}[1,579]=102,9 ; p<0,001\right)$.

\section{Diskussion} len Erschöpfung. Bei der Einbeziehung
Das Hauptziel dieser Studie bestand in der Überprüfung der statistischen Zusammenhänge von arbeitsbezogenen Faktoren, emotionaler Erschöpfung, emotionaler Kompetenz (Regulation) 


\begin{tabular}{|c|c|c|c|c|c|c|c|c|}
\hline Variablen & 1 & 2 & 3 & 4 & 5 & 6 & 7 & 8 \\
\hline \multicolumn{9}{|l|}{ Abhängige Variable } \\
\hline 1 Wohlbefinden & 1 & - & - & - & - & - & - & - \\
\hline \multicolumn{9}{|l|}{ Unabhängige Variablen } \\
\hline 2 Arbeitsintensität & $-0,31^{b}$ & 1 & - & - & - & - & - & - \\
\hline 3 Spielräume & $0,27^{b}$ & $-0,25^{b}$ & 1 & - & - & - & - & - \\
\hline $\begin{array}{l}4 \text { Emotionale Erschöp- } \\
\text { fung }\end{array}$ & $-0,45^{b}$ & $0,48^{b}$ & $-0,16^{b}$ & 1 & - & - & - & - \\
\hline 5 Regulieren & $0,37^{b}$ & $-0,15^{b}$ & $0,08^{a}$ & $-0,32^{b}$ & 1 & - & - & - \\
\hline \multicolumn{9}{|l|}{ Kontrollvariable } \\
\hline 6 Geschlecht & $-0,07$ & $0,17^{b}$ & 0,04 & $0,23^{b}$ & $-0,10^{\mathrm{a}}$ & 1 & - & - \\
\hline 7 Alter & $0,09^{\mathrm{a}}$ & $0,18^{b}$ & $0,16^{\mathrm{b}}$ & $0,15^{b}$ & 0,05 & $0,23^{b}$ & 1 & - \\
\hline 8 Ursprungsdatensätze & $-0,02$ & $-0,20^{b}$ & $-0,20^{b}$ & $-0,35^{\mathrm{b}}$ & $0,13^{b}$ & $-0,55^{b}$ & $-0,34^{b}$ & 1 \\
\hline \multicolumn{9}{|c|}{$\begin{array}{l}n=602-620 \\
n \text { Stichprobengröße, } p \text { Wahrscheinlichkeiten } \\
{ }^{*} p<0,05,{ }^{* * *} p<0,01\end{array}$} \\
\hline
\end{tabular}

Tab. 2 Hierarchische Regression: Einfluss der psychischen Arbeitsmerkmale, der emotionalen Erschöpfung, der Regulation von Emotionen auf das psychische Wohlbefinden

\begin{tabular}{|c|c|c|c|c|}
\hline \multirow[b]{2}{*}{ Schritte } & & \multicolumn{3}{|c|}{ WHO5 } \\
\hline & & B & SE B & $\beta$ \\
\hline \multirow[t]{5}{*}{1} & Konstante & 2,40 & 0,15 & - \\
\hline & Geschlecht & 0,30 & 0,12 & 0,13 \\
\hline & Alter & 0,00 & 0,00 & 0,10 \\
\hline & Ursprungsdatensätze & $-0,14$ & 0,13 & $-0,06$ \\
\hline & - & - & $\begin{array}{l}R^{2}=0,02^{*} \\
F=3,63^{*}\end{array}$ & $R^{2}$ korr $=0,01$ \\
\hline \multirow[t]{2}{*}{2} & Arbeitsintensität & $-0,47$ & 0,05 & $-0,34$ \\
\hline & - & - & $\begin{array}{l}R^{2}=0,13^{* * *} \\
F=73,31^{* * *}\end{array}$ & $\mathrm{R}^{2}$ korr $=0,12$ \\
\hline \multirow[t]{2}{*}{3} & Spielräume & 0,22 & 0,05 & 0,17 \\
\hline & - & - & $\begin{array}{l}R^{2}=0,15^{* * *} \\
F=17,23^{* * *}\end{array}$ & $\mathrm{R}_{\text {korr }}=0,15$ \\
\hline \multirow[t]{2}{*}{4} & Emotionale Erschöpfung & $-0,32$ & 0,03 & $-0,43$ \\
\hline & - & - & $\begin{array}{l}\mathrm{R}^{2}=0,28^{* * *} \\
F=102,9^{* * *}\end{array}$ & $\mathrm{R}^{2}$ korr $=0,27$ \\
\hline \multirow[t]{2}{*}{5} & Emotionsregulation & 0,36 & 0,05 & 0,25 \\
\hline & - & - & $\begin{array}{l}R^{2}=0,34^{* * *} \\
F=47,2^{* * *}\end{array}$ & $R_{\text {korr }}^{2}=0,33$ \\
\hline \multicolumn{5}{|c|}{$\begin{array}{l}\text { WHO5: Psychisches Wohlbefinden gemessen mit WHO5 Fragebogen. Kontrollvariablen: Geschlecht, } \\
\text { Alter, Ursprungsdatensätze. Alle Regressionskoeffizienten wurden dem letzten } S c h r i t t \text { der Regressi- } \\
\text { onsgleichung entnommen. Für den ersten } S c h r i t t ~ d f 1=3, d f 2=582 \text {, für den zweiten } S c h r i t t ~ d f 1=1 \text {, } \\
d f 2=581 \text {, für den dritten } S c h r i t t ~ d f 1=1, d f 2=580, \text { für den vierten } S c h r i t t ~ d f 1=1, d f 2=579 \text {, für den } \\
\text { fünften } S c h r i t t ~ d f=1, d f 2=578 \\
B \text { Regressionsgewicht, } S E B \text { Standardfehler von } B, \beta \text { standardisiertes Regressionsgewicht, } R^{2} \text { Regressi- } \\
\text { onskoeffizient, } F \text { Anteil der erklärten Varianz im Gesamtmodell } \\
\text { Signifikanz (einseitig): }{ }^{*} p<0,5,{ }^{* * *} p<0,01\end{array}$} \\
\hline
\end{tabular}

und psychischem Wohlbefinden unter Einbeziehung von Geschlecht, Alter und den ursprünglichen Einzeldatensätzen aus der Altenpflege und dem Rettungsdienst. Außerdem prüfte die Studie den statischen Aufklärungsbeitrag der genannten Konstrukte auf das psychische Wohlbefinden. Die Studie liefert neue Erkenntnisse zu Zusammenhängen zwischen dem Umgang mit eigenen Emotionen und emotionaler Erschöpfung als der Kerndimension von Burnout bei einer gesunden Stichprobe in Gesundheitsberufen. Insbesondere die emotionale Erschöpfung zeigt sich als ein starker Risikofaktor in Bezug auf das psychische Wohlbefinden. Aber auch die subjektive Einschätzung von Arbeitsbedingungen (Arbeitsintensität und Spielräume) und die positive gezielte Beeinflussung der eigenen Emotionen als Regulierungsstrategie leisten einen wichtigen Beitrag für die Vorhersage der psychischen Gesundheit.

Hinsichtlich des psychischen Wohlbefindens ist festzustellen, dass 55,6\% der Beschäftigten in den beiden ausgewählten Gesundheitsberufen ein mittleres bis hohes Wohlbefinden berichten. Allerdings gaben auch $43,1 \%$ ein niedriges Wohlbefinden an. Besonders bei der Altersgruppe bis 24 Jahren zeigt sich mit $51 \%$ angegebenem niedrigem Wohlbefinden eine auffällige Tendenz der überwiegend in dieser Altersklasse männlichen Teilnehmer (57,2\%). Dazu konform berichten die Beschäftigten eine eher unterdurchschnittliche Ausprägung der Kompetenz, ihre eigenen Gefühle gezielt $\mathrm{zu}$ regulieren. Vorangegangene Studien belegen bereits einen Zusammenhang des Wohlbefindens mit den emotionalen Kompetenzen [5,9] und dessen direkte positive Beeinflussung durch den Einsatz des TEK. Hier konnte im Prä-Post-Vergleich mit einer Kontrollgruppe nachgewiesen werden, dass ein positiver statistischer mittlerer $\mathrm{Zu}$ sammenhang zwischen der emotionalen Kompetenz (Regulation) und dem psychischen Wohlbefinden besteht $(r=42$; ${ }^{*} p<0,05$; [9]). Somit stellt die Stärkung emotionaler Kompetenzen eine wichtige personale Ressource dar, welche sich in eine Reihe bereits erfolgreicher Stressmanagement-Interventionen einreiht.

Die Beschäftigten in der stationären Altenpflege und im Rettungsdienst berichten darüber hinaus über unterschiedliche Ausprägungen der emotionalen Erschöpfung. Im Durchschnitt lag eine mittlere Erschöpfung vor, wobei $18 \%$ sehr hohe Werte angaben. Auch hier konnte bei Beschäftigten der Altenpflege durch das TEK ein signifikanter negativer Zusammenhang zwischen der emotionalen Kompetenz (Regulation) und emotionaler Erschöpfung als Trainingsgewinn $\left(r=-0,31 ;{ }^{* *} p<0,01 ;[12]\right)$ gefunden werden. Demnach geben die 
angegebenen Werte der emotionalen Erschöpfung bereits bei der deskriptiven Datenanalyse einen Hinweis auf einen möglichen Zusammenhang mit der emotionalen Kompetenz.

Bezogen auf die psychische Belastung ist festzustellen, dass die Beschäftigten in den ausgewählten Gesundheitsberufen Arbeitsintensität und Spielräume im mittleren Bereich einschätzen. Die Angaben der Arbeitsintensität sind sicher dem Umstand geschuldet, dass insbesondere die Stichprobe der Rettungskräfte überwiegend aus Auszubildenden bestand. Dem gegenüber berichtet eine Rettungsdienststudie [21] von hohen Fehlbeanspruchungen durch Schichtarbeit, fehlendes Personal, Überstunden und Zeitdruck.

Aus den Prüfungen der korrelativen Zusammenhänge bestätigen sich die deskriptiven Ergebnisse. Es zeigt sich v.a. eine signifikante Korrelation von Wohlbefinden mit emotionaler Erschöpfung, Arbeitsintensität und dem Regulieren der eigenen Emotionen im mittleren Bereich. Dass sich die quantitativen Arbeitsanforderungen, aber auch die Regulation der Emotionen im Sinne einer kognitiv-psychischen Handlungsregulation, auf das psychische Erleben auswirken, konnte bereits in etablierten Studien bestätigt werden [20,29]. Ebenso existieren bereits empirische Befunde für einen Zusammenhang von hinderlichen Regulierungsstrategien und erhöhter emotionaler Erschöpfung [1, 16, 26].

Die ermittelten Ergebnisse der Regressionsanalyse bestätigen frühere Studien, nach denen ER-Kompetenzen positiv mit der psychischen Gesundheit zusammenhängen. Die Studie konnte zeigen, dass die Regulation der eigenen Emotionen neben den erhobenen Arbeitsmerkmalen und der emotionalen Erschöpfung das Wohlbefinden direkt beeinflusst. Insgesamt konnten die in der Analyse berücksichtigten Variablen unter Einbeziehung der Kontrollvariablen das subjektive Wohlbefinden zu $33 \%$ erklären. Natürlich gibt es darüber hinaus etliche berufliche und private Einflüsse, die mitbestimmen, wie wohl sich jemand fühlt. Beispielhaft können der körperliche Gesundheitszustand, Entwicklungsmöglichkeiten bei der Ar- beit, die Qualität der sozialen Kontakte und Beziehungen bei und außerhalb der Arbeit neben vielen weiteren Faktoren angeführt werden. Angesichts dieser Tatsache sind $30 \%$ eine durchaus beachtliche Größe. Die ermittelten Ergebnisse bestätigen frühere Beobachtungen, nach denen die Art, wie Personen ihre Gefühle regulieren, das psychische Befinden beeinflusst [3, 4]. Es konnten Zusammenhänge mit emotionaler Erschöpfung und Wohlbefinden nachgewiesen werden. Dieser Befund ist an sich nicht neu, da bereits zahlreiche Studien vergleichbare Beziehungen aufzeigten [20, 23]. Bisher wurde die ER-Kompetenz dabei jedoch selten als eine allgemeine Fähigkeit aufgefasst, die verschiedene Ausprägungen aufweisen kann und zwischen Personen variiert. Allerdings können die unterschiedlichen Emotionsregulationsstrategien (z.B. Oberflächen- oder Tiefenhandeln) nicht per se in gesundheitsschützend oder -beeinträchtigend unterteilt werden. Wie genau sie sich letztlich auf das Befinden auswirken, hängt vielmehr vom jeweiligen Anwendungskontext, den Dimensionen von Emotionen (geforderte, die gefühlte und die gezeigte Emotion) und illegitimen Stressoren ab [27, 28, 30]. Das flexible Anpassen der für die Situation am besten geeigneten Strategie scheint im Hinblick auf eine gesundheitsfördernde Emotionsregulation ausschlaggebend $\mathrm{zu}$ sein [28]. Ist dieses gut ausgeprägt, modifizieren Personen ihre Gefühle derart, dass sie häufig gar nicht erst den ungünstigen Zustand emotionaler Dissonanz erleben.

Zukünftige Erhebungsinstrumente sollten Probanden deshalb danach fragen, wie effektiv sie ihre Emotionen i. Allg. anpassen. Erfasst man die ERKompetenz als interindividuell unterschiedlich ausgeprägtes Fähigkeitsmerkmal, lassen sich die Ergebnisse von Interventionsstudien besser beurteilen und gezielt verhaltensbezogene Maßnahmen in der Primärprävention ableiten. Als Erhebungsinstrumente empfehlen sich neben dem in dieser Studie verwendeten SEK-27 [6, 27] die Difficulties in Emotion Regulation Scale [18] oder die Emotion Reactivity Scale [24].

\section{Fazit für die Praxis}

- Sowohl Eigenschaften und Fähigkeiten der Person als auch Merkmale des Arbeitskontextes (Arbeitsintensität und Spielräume) bestimmen, wie gesund sich Beschäftigte mit ständigen Interaktionen bei ihrer Arbeit fühlen.

- Persönliche emotionale (ER-)Kompetenzen sind ein wichtiger Faktor für die psychische Gesundheit. Mit zunehmenden ER-Kompetenzen fühlen sich die befragten Mitarbeiter weniger emotional erschöpft und berichten ein höheres Wohlbefinden.

- Stärkung der emotionalen Kompetenzen durch spezielle Trainings, die den konstruktiven Umgang mit emotionalen Belastungen vermitteln und die Beschäftigten dabei unterstützen, funktionellere Emotionsregulationsstrategien zu festigen und Gefühle flexibler zu regulieren.

- Verringerung der Arbeitsintensität, z. B. durch angemessene Personalbesetzung bzw. Betreuungsschlüssel, fähigkeitsgerechte Aufgabenzuweisung.

- Erweiterung des Handlungs- und Entscheidungsspielraums, z. B. durch weniger strenge Vorgaben hinsichtlich darzustellender Gefühle im Umgang mit Patienten, Mitbestimmung bei der Arbeitsteilung mit den Kollegen, eine flexible Gestaltung von Schicht- und Einsatzplänen.

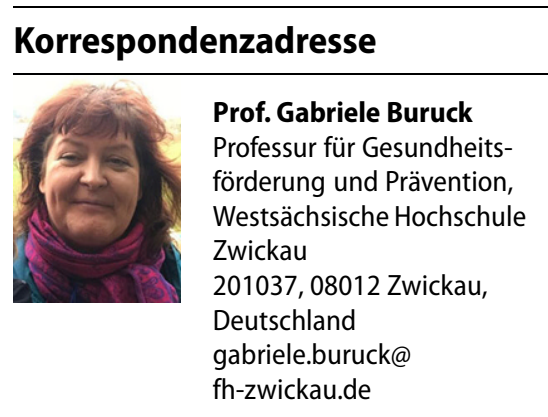

Funding. Open Access funding enabled and organized by Projekt DEAL.

\section{Einhaltung ethischer Richtlinien}

Interessenkonflikt. G. Buruck und S. Haitsch geben an, dass kein Interessenkonflikt besteht. 
Für diesen Beitrag wurden von den Autoren keine Studien an Menschen oder Tieren durchgeführt. Für die aufgeführten Studien gelten die jeweils dort angegebenen ethischen Richtlinien.

Open Access. Dieser Artikel wird unter der Creative Commons Namensnennung 4.0 International Lizenz veröffentlicht, welche die Nutzung, Vervielfältigung Bearbeitung, Verbreitung und Wiedergabe in jeglichem Medium und Format erlaubt, sofern Sie den/die ursprünglichen Autor(en) und die Quelle ordnungsgemäß nennen, einen Link zur Creative Commons Lizenz beifügen und angeben, ob Änderungen vorgenommen wurden.

Die in diesem Artikel enthaltenen Bilder und sonstiges Drittmaterial unterliegen ebenfalls der genannten Creative Commons Lizenz, sofern sich aus der Abbildungslegende nichts anderes ergibt. Sofern das betreffende Material nicht unter der genannten Creative Commons Lizenz steht und die betreffende Handlung nicht nach gesetzlichen Vorschriften erlaubt ist, ist für die oben aufgeführten Weiterverwendungen des Materials die Einwilligung des jeweiligen Rechteinhabers einzuholen.

Weitere Details zur Lizenz entnehmen Sie bitte der Lizenzinformation auf http://creativecommons.org/ licenses/by/4.0/deed.de.

\section{Literatur}

1. Aldao A, Nolen-Hoeksema S, Schweizer S (2010) Emotion-regulation strategies across psychopathology: a meta-analytic review. Clin Psychol Rev $30(2): 217-237$

2. Berking $M$ (2017) Training emotionaler Kompetenzen, 4. Aufl. Springer, Berlin Heidelberg

3. Berking M, Ebert D, Cuijpers P et al (2013) Emotion regulation skills training enhances the efficacy of inpatient cognitive behavioral therapy for major depressive disorder: a randomized controlled trial. Psychother Psychosom 82(4):234-245

4. Berking M, Wirtz CM, Svaldi J et al (2014) Emotion regulation predicts symptoms of depression over five years. Behav Res Ther 57:13-20

5. Berking M, Wupperman P (2012) Emotion regulation and mental health: recent findings, current challenges, and future directions. Curr Opin Psychiatry 25:128-134

6. Berking M, Znoj H (2008) Entwicklung und Validierung eines Fragebogens zur standardisierten Selbsteinschätzung emotionaler Kompetenzen (SEK-27). Z Klin Psychol Psychopathol Psychother 56(2):141-153

7. Buruck G, Brom SS, Kotte H, Richter P (2015) Evaluierung von Gesundheitspräventionen in der stationären Altenpflege. In: Badura B, Ducki A, Schröder H, Klose J, Meyer M (Hrsg) FehlzeitenReport 2015. Springer, Berlin, Heidelberg, S 85-95

8. Buruck G, Dörfel D (2018) iga.Report 37. Emotionsregulation in der Arbeit am Beispiel Rettungsdienst, 1. Aufl. iga, Dresden

9. Buruck G, Dörfel D, Kugler J et al (2016) Enhancing well-being at work: The role of emotion regulation skills as personal resources. JOccup Health Psychol 21(4):480-493

10. Buruck G, Melzer M (2019) Emotionale Arbeitsanforderungen und psychische Gesundheit. In: Knieps F, Pfaff H (Hrsg) BKK Gesundheitsreport 2019: Psychische Gesundheit und Arbeit Zahlen, Daten, Fakten. MWV, Berlin, S223-230
11. Buruck G, Tomaschek A (2018) Konstruktiver Umgang mit Emotionen und Verringerung von Burnout - Beispiel einer erfolgreichen Intervention in einer Hochrisikoberufsgruppe. Wirtschaftspsychologie 1:17-51

12. Clauß G, Finze F-R, Partzsch L (1999) Grundlagen, 3. Aufl. Statistik für Soziologen, Pädagogen, Psychologen und Mediziner, Bd. 1. Harri Deutsch, Thun

13. Cohen J (1992) Statistical power analysis. Curr Dir Psychol Sci 1(3):98-101

14. Eid M, Gollwitzer M, Schmitt M (2013) Statistik und Forschungsmethoden, 3. Aufl. Beltz, Weinheim, Basel

15. Gößwald A, Lange M, Kamtsiuris Petal (2012)DEGS: Studie zur Gesundheit Erwachsener in Deutschland. Bundesgesundheitsblatt 55:775-780

16. Grandey AA, Frone MR, Melloy RCetal (2019) When are fakers also drinkers? A self-control view of emotional labor and alcohol consumption among U.S. service workers. J Occup Health Psychol 24(4):482-497

17. Grandey AA, Melloy RC (2017) The state of the heart: Emotional labor as emotion regulation reviewed and revised. J Occup Health Psychol 22(3):407-422

18. Gratz KL, Roemer L (2004) Multidimensional assessment of emotion regulation and dysregulation: development, factor structure, and initial validation of the difficulties in emotion regulation scale. J Psychopathol Behav Assess 26(1):41-54

19. Hacker W (2020) Psychische Regulation von Arbeitstätigkeiten 4.0.vdf, Zürich

20. Hulsheger UR, Schewe AF (2011) On the costs and benefits of emotional labor: a meta-analysis of three decades of research. J Occup Health Psychol 16:361-389

21. Karutz H, Overhagen M, Stum J (2013) Psychische Belastungen im Wachalltag von Rettungsdienstmitarbeitern und Feuerwehrleuten. Präv Gesundheitsf 8(3):204-211

22. Korczak D, Huber B, Kister C (2010) Differential diagnostic of the burnout syndrome. GMS Health Technol Assess 6:Doc9

23. Mesmer-Magnus JR, DeChurch LA, Wax A (2012) Moving emotional labor beyond surface and deep acting: a discordance-congruence perspective. Organ Psychol Rev 2(1):6-53

24. Nock MK, Wedig MM, Holmberg EB et al (2008) The emotion reactivity scale: development, evaluation, and relation to self-injurious thoughts and behaviors. Behav Ther 39(2):107-116

25. Scheibe S (2019) Predicting real-world behaviour: Cognition-emotion links across adulthood and everyday functioning at work. Cogn Emot 33:126-132

26. Schöllgen I,SchulzA (2016) Psychische Gesundheit in der Arbeitswelt - Emotionsarbeit, 1. Aufl. Bundesanstalt für Arbeitsschutz und Arbeitsmedizin, Dortmund

27. Semmer NK, Jacobshagen N, Keller AC et al. (2020) Adding insult to injury: Illegitimate stressors and their association with situational well-being, social self-esteem, and desire for revenge. Work \& Stress. https://doi.org/10.1080/02678373.2020.1857465

28. Troy AS, Shallcross AJ, Mauss IB (2013) A person-bysituation approach to emotion regulation: cognitive reappraisal can either help or hurt, depending on the context. Psychol Sci 24(12):2505-2514

29. Zapf D, Holz M (2006) On the positive and negative effects of emotion work in organizations. Eur J Work Organ Psychol 15:1-28

30. ZapfD, Kern M, Tschan F et al (2021) Emotion Work: A Work Psychology Perspective. Annu Rev Organ Psychol Organ Behav 8(1):139-172 